\title{
The 3E's of Sustainability Establishing a Measure of the Success Rate of Urban Smart Growth
}

\author{
Jixian Wang \\ School of North China Electric Power University, Baoding 071000, China \\ 939189710@qq.com
}

Abstract: In order to achieve sustainable development planning goals, many cities are implementing smart growth plans. We use the 3E's objective of sustainable development as the basis for analysis. And we establish model to make a comprehensive evaluation.and we define the metric of success rate of urban smart growth, thus we get the evaluation standard.

Keywords: Urban smart growth, Sustainable Cities, the 3E's of sustainability, Principal Component Analysis

\section{Introduction}

Since the 20th century the 60's, with the deepening of urban suburbanization, the review of 'Urban Sprawl'has attracted more and more attention from the public. 'Urban Sprawl'mainly refers to the urban space of low-density expansion. In this process involved agricultural land ,the transformation of open space to construction land, and with a series of economic, social and environmental problems 。 Cities are the main bearers of the future population and economy, representing the occupation of land, energy consumption, resulting in the main environmental pressure. It is estimated that by 2050 , the urban population will reach $66 \%$ of the world's total population.

Smart growth is a kind of urban planning theory originated from the 1990s, that means under resource constraints, through the realization of social, environmental, and economic balanced development, Optimize the urban structure. Its goal is to curb the continued urban sprawl and the loss and reduction of urban-centered surrounding farmland, which is a city growth policy to address urban sprawl。Therefore, the sustainable construction of the city becomes more and more important. [1,3,4]

\section{Model assumptions}

We need to choose two cities which both the population is 10 million to 50 million in different continents Next, we make the following assumptions to define an metric that measures the success rate of urban growth.

(1) Ignore the impacts of the extreme disasters.

(2) Ignore the impacts of the migration of population

\section{Factors selection}

we describe and analyze the factors that affect this growth from different development perspectives, and use the following metric to evaluate the degree of the development.

Table 1 City smart growth evaluation of the indicators

\begin{tabular}{|c|c|l|}
\hline level I indicator & Level II indicators & Level III indicators \\
\hline \multirow{3}{*}{$\begin{array}{c}\text { To achieve smart } \\
\text { growth in the city }\end{array}$} & \multirow{3}{*}{ Economic prosperity } & Per capita GDP $\left(x_{1}\right)$ \\
\cline { 2 - 3 } & Social equality & The total retail sales of social consumer goods $\left(x_{2}\right)$ \\
\cline { 2 - 3 } & unemployment rate $\left(x_{3}\right)$ \\
\cline { 2 - 3 } & $\begin{array}{c}\text { Environmental } \\
\text { sustainability }\end{array}$ & Per coefficient $\left(x_{4}\right)$ \\
\cline { 2 - 3 } & Unit GDP energy consumption $\left(x_{6}\right)$ \\
\hline
\end{tabular}




\section{Model establishment}

\section{- Model principle}

(1) the value of the $j$ index of the $i$ evaluation object is $a_{i j}$. Each metric value is converted into a standardized index,

$$
\begin{aligned}
& \tilde{x}_{\mathrm{ij}}=\frac{\mathrm{x}_{\mathrm{ij}}-\mathrm{u}_{\mathrm{j}}}{\mathrm{s}_{j}},(\mathrm{i}=1,2, \ldots \mathrm{n} ; \mathrm{j}=1,2, \ldots \mathrm{m}) \\
& \text { among them, } u_{j}=\frac{1}{n} \sum_{i=1}^{n} x_{i}, s_{j}=\frac{1}{n-1} \sum\left(x_{i j}-u_{j}\right)^{2}
\end{aligned}
$$

After the value of each influencing factor comes in, we can get $\tilde{x}_{i j}$, is the standardized indicator variable.

(2) Calculate the correlation coefficient matrix $R=\left(r_{i j}\right)_{m * n}$

(3) Calculate eigenvalues and eigenvectors

Calculate the eigenvalues of the correlation coefficient matrix $\lambda_{1} \geq \lambda_{2} \geq \cdots \lambda_{m} \geq 0$, and the corresponding eigenvectors $\mu_{1}, \mu_{2, \ldots} \mu_{m}$, among them $\left(\mu_{1 j}, \mu_{2 j, \ldots,} \mu_{m j}\right)^{T}$, the new $m$ variables are composed of eigenvectors

$$
\left\{\begin{array}{c}
y_{1}=\mu_{11} \tilde{x}_{1}+\mu_{21} \tilde{x}_{2}+\cdots \mu_{m 1} \tilde{x}_{m} \\
y_{2}=\mu_{12} \tilde{x}_{1}+\mu_{22} \tilde{x}_{2}+\cdots \mu_{m 2} \tilde{x}_{m} \\
\ldots \cdots \cdots \cdots \cdots \cdots \cdots \cdots \cdots \cdots \cdots \cdots \cdots \\
y_{m}=\mu_{1 m} \tilde{x}_{1}+\mu_{2 m} \tilde{x}_{2}+\cdots \mu_{m m} \tilde{x}_{m}
\end{array}\right.
$$

In the formula, $y_{1}$ is the first principal component, $y_{2}$ is the second principal component ,..., $y_{m}$ are the $m$ principal component

(4) Select $p(p \leq m)$ principal component, calculate the comprehensive evaluation value

Calculating eigenvalues $\lambda_{j}(j=1,2, \ldots, m)$ 's contribution rate of information. Namely,

$$
\mathrm{b}_{j}=\frac{\lambda_{j}}{\sum_{k=1}^{m} \lambda_{k}},(j=1,2, \ldots, m),
$$

is the main ingredient $y_{j}$ 's contribution rate of information

$$
\alpha_{p}=\frac{\sum_{k=1}^{p} \lambda_{k}}{\sum_{k=1}^{m} \lambda_{k}}
$$

is the main ingredient, the main ingredient $\alpha_{p}$ 's cumulative contribution rate, when $\alpha_{p}$ is close to $1\left(\alpha_{p}=0.85,0.90,0.95\right)$, Then select the top $p$ principal components to analysis .

Calculate the composite score

$$
\mathrm{Z}=\sum_{j=1}^{p} b_{j} y_{j}
$$

Among them, $b_{j}$ is the $j$ contribution of Principal Component to Information, the evaluation can be carried out on the basis of the integrated score value. [2]

- Definition of smart growth success rate

According to the principles of our model, when we bring in the data factors after dimension reduction, we can obtain the city's comprehensive score $Z(t), Z(t)-Z(t-1)$ rates this city's Smart Growth value, taking into account the side effects of population growth and urban growth on smart growth, next,we set the current population growth rate for the year is $P$, The annual growth rate of urban area is $Q$, We define the $t$ year Smart Growth success rate is $W(t)$ then we can get: 


$$
W(t)=\frac{Z(t)-Z(t-1)}{(1+\alpha P)(1+\beta Q)}
$$

Among them, $\alpha$ is the annual population growth rate adjustment system, $\beta$ is the annual growth rate of urban area adjustment factor.

- Standard division

Through the data processing, we divided the following criteria to determine the extent of the city's smart growth

Table 2. The classificatory standard

\begin{tabular}{cl|c|c}
\hline \multicolumn{2}{c|}{ level } & W & Smart growth situation \\
\hline I excellent & $>3.8$ & To meet the requirements of smart growth \\
\hline II good & $3.6 \sim 3.8$ & $\begin{array}{l}\text { the success rate of smart growth basically meet the } \\
\text { requirements, there can be improved }\end{array}$ \\
\hline III medium & $3.4 \sim 3.6$ & There are many problems in Urban Smart Growth \\
\hline IV poor & $<3.4$ & Urban smart growth is poor \\
\hline
\end{tabular}

\section{Conclusions}

Smart growth focused on urban construction particularly emphasis on sustainable economies.we can provide a simple idea for the construction of urban smart growth. "Smart growth" is a comprehensive strategy that covers the principles of urban development at multiple levels.

We use Principal Component Analysis to obtain the main components, which reduces the factors which needed to consider. And using the principal component analysis method to obtain the comprehensive score as a city comprehensive score, at the same time, with the urban development of the overall score of the increase in the amount of time as the city smart growth score. Fully explain the growth of this change in indicators. Not only for the development of the same size of the city, for the development of different sizes of cities can also be compared.

When defining the success rate of smart growth of a city, we take into account that urban growth scores and urban area and population growth ,which makes the results more accurate.

\section{References}

[1] Cheng Maoji. Research on the evaluation and optimization of urban growth in Nanjing based on the perspective of smart growth. Diss. Nanjing Normal University, 2012

[2] Shoukui si. Mathematical Modeling Algorithms and Applications. National Defense Industry Press, 2015

[3] https://smartgrowthamerica.org/

[4] https://www.epa.gov/smartgrowth/smart-growth-publicatio 